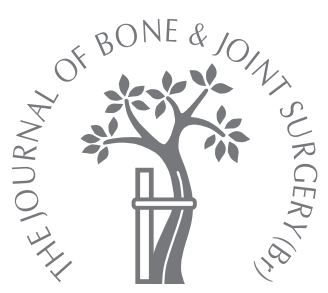

G. Hooper,

A. Rothwell,

C. Frampton

From Christchurch

Public Hospital,

Christchurch, New

Zealand
G. Hooper, FRACS, Associate

Professor, Head of Department

A. Rothwell, FRACS,

Emeritus Professor

C. Frampton, $\mathrm{PhD}$

Statistician

Department of Orthopaedic

Surgery

Christchurch Public Hospital

Private Bag, Christchurch, New

Zealand.

Correspondence should be sent to Mr G. Hooper at Leinster

Orthopaedic Centre, 151

Leinster Road, Christchurch,

New Zealand; e-mail:

ghooper@leinsterortho.co.nz

(C)2009 British Editorial Society

of Bone and Joint Surgery

doi:10.1302/0301-620X.91B1.

$20484 \$ 2.00$

$J$ Bone Joint Surg $[\mathrm{Br}]$ 2009;91-B:58-63.

Received 13 November 2007;

Accepted after revision 27 June 2008

\title{
The low contact stress mobile-bearing total knee replacement
}

\author{
A PROSPECTIVE STUDY WITH A MINIMUM FOLLOW-UP OF TEN \\ YEARS
}

We have examined the outcome of 400 consecutive patients who underwent total knee replacement with the Low Contact Stress mobile-bearing system between 1993 and 1994 and were followed up for a minimum of ten years. All operations were performed by surgeons in Christchurch, New Zealand, who used no other knee prosthesis during the study time.

At ten years after operation 238 patients ( 244 knees) remained for independent clinical and radiological assessment. There was a significant improvement $(p<0.001)$ in the postoperative knee scores at one, three, seven and ten years, although a slight deterioration in the score occurred between seven and ten years which did not reach statistical significance. The survival for polyethylene wear or loosening was $97 \%(95 \% \mathrm{Cl} 96$ to 98 ) and survival using reoperation for any cause was $92 \%(95 \% \mathrm{Cl} 90$ to 94$)$ at 12 years. Polyethylene wear was more common in the meniscal-bearing component, with five knees requiring revision and a further eight demonstrating early wear. Osteolysis was not seen in the rotating platform component, but was present in three of the meniscal-bearing knees.

There was no association between the radiological alignment at one year and those knees that subsequently required revision. However, there was an association between the overall limb alignment and the Western Ontario McMasters University score $(p<0.001)$.

The Low Contact Stress mobile-bearing total knee replacement has proved to be a reliable implant at ten years when used in primary knee replacement irrespective of the deformity and diagnosis.

Total knee replacement (TKR) using fixedbearing implants has produced good long-term survival of up to $97 \%$ at ten years. ${ }^{1-6}$ However, there have been problems with premature polyethylene wear and aseptic loosening with some implants. Our experience with fixedbearing prostheses in the 1980s revealed early wear, particularly with a thin $(6 \mathrm{~mm})$ polyethylene component, and a high incidence of fracture of the tibial tray. ${ }^{7}$ This has subsequently been confirmed in other studies. ${ }^{8}$ These failures prompted our department to change the prostheses we used to the Low Contact Stress mobile-bearing TKR (DePuy, Leeds, United Kingdom).

The Low Contact Stress mobile-bearing TKR was introduced to improve the congruity between the femoral component and the polyethylene insert throughout knee movement, with the intention of limiting the stress across the bone-implant interface. The mobile polyethylene insert, in the form of either twin meniscal bearings or a single rotating platform, permitted increased congruity, particularly through the first $40^{\circ}$ of knee flexion, while at the same time protecting the implant from excessive shear stresses across the implantbone interface. The early results from the designing surgeon were encouraging, with outcomes at least as good as the early results of fixed-bearing TKR. ${ }^{9}$

From 1990, we have used the Low Contact Stress mobile-bearing TKR as the only primary prosthesis in a population of approximately 400 000. In 1993 we initiated a prospective study of this prosthesis and now report our minimum ten-year results, with particular emphasis on the survival of the implant and the radiological incidence of polyethylene wear and component loosening. Additionally, we wished to assess the clinical outcome using current scoring systems to enable comparison with fixed-bearing TKRs. Our hypothesis was that the revision rate due to polyethylene wear and component loosening would be diminished in comparison with fixed-bearing prostheses using the Low Contact Stress mobilebearing arthroplasty. 


\section{Patients and Methods}

Ethical approval was obtained from the Christchurch Hospital Ethics Committee, and all patients gave informed consent before entering the study.

Commencing in October 1993, we continued to enrol patients until 400 had been recruited (December 1994), of whom 14 underwent bilateral procedures. All 14 orthopaedic surgeons who performed TKR in our city contributed to the study, and the patients were entered consecutively. All the surgeons were general orthopaedic surgeons, with none specialising in TKR or knee surgery. During this period all knees were replaced using the Low Contact Stress TKR system for which polyethylene components were sterilised by irradiation in air. No patient was excluded because of the severity of their disease or deformity.

The manual alignment instruments supplied by the company (DePuy, Leeds, United Kingdom) were used for the procedure, although each surgeon adhered to his own routine practice and no attempt was made to standardise the procedure. All surgeons used a high thigh tourniquet, which was generally released following skin closure. Most patients had a spinal anaesthetic and used thromboembolic stockings, but no routine chemical thromboprophylaxis was used. High-risk patients were given low molecular weight heparin peri-operatively and warfarin post-operatively from one to six weeks. All patients had peri-operative antibiotics until the drains were removed, 24 hours post-operatively and were mobilised the day after surgery.

An independent research assistant $(\mathrm{TH})$ recorded all preand post-operative data. The Knee Society pain and function score,${ }^{10}$ the New Jersey Knee Score ${ }^{11}$ and the Western Ontario and McMaster Universities Osteoarthritis score (WOMAC) $^{12}$ were recorded pre-operatively and again at one, three and seven years. The WOMAC score was recorded again at ten years.

All radiographs were performed at one, three and ten years according to the Knee Society guidelines, ${ }^{13}$ but were modified to include standard weight-bearing anteroposterior (AP) and lateral radiographs with the patella directed forwards. All skyline patellar views were taken in approximately $30^{\circ}$ of flexion with the knee unloaded. Patellar subluxation was measured by using the skyline view and comparing the position of the centre of the patella to the centre of the femoral groove. ${ }^{14}$ All radiographs were assessed using the Knee Society ${ }^{13}$ rating system by a single examiner $(\mathrm{GH})$, who was blinded to both the clinical outcome and the surgeon involved. All radiographs were digitised and measured manually using a ruler and goniometer on the radiographs at a single sitting.

Statistical method. A power analysis at the time of the study design, using existing data, indicated that with 400 patients there would be sufficient power to define the revision rate for aseptic loosening and polyethylene wear with a $95 \%$ confidence interval $(\mathrm{CI})$ of approximately $2 \%$ for up to 20 years.
A paired $t$-test was used to compare pre- and postoperative scores, and the associations between radiological parameters and functional outcome measures were tested using Pearson's correlation coefficient.

A Kaplan-Meier ${ }^{15}$ survival analysis was conducted on the sample using reoperation for any cause as the endpoint. Survival analysis was also performed using revision due to polyethylene wear and loosening as the criteria.

Statistical significance was assumed with a two-tailed p-value $<0.05$.

Validation of revision data. We used the New Zealand Joint Registry ${ }^{16}$ to validate the records of revision surgery within New Zealand, excluding the Christchurch area, to identify any patients revised outside our unit. This registry records all primary and revision joint replacements performed in New Zealand, and has a $98 \%$ compliance rate. ${ }^{16}$

\section{Results}

Of the initial study group, five patients (five knees) were excluded because of errors in documentation and a further 24 (24 knees) were excluded because revision Low Contact Stress implants were used. By ten years, 109 patients had died and a further 24 had withdrawn from the study because they had either moved out of the area or emigrated, leaving 238 patients ( 244 knees) available for review.

Of the initial study group there were 25 patients ( 25 knees, $10.5 \%$ ) who required a further operation during this period, and although their results have been included they will be discussed separately. Detailed clinical results were available for all of these patients.

Clinical and radiological examination was completed on 186 patients (192 knees in 105 men and 81 women), all of whom underwent WOMAC assessment at a minimum of ten years. The remaining 52 patients ( 52 knees), who could not be reviewed at ten years, were either medically infirm or confined to a rest home with limited functional requirements. Where possible, these patients were requested to complete a WOMAC questionnaire either by telephone interview or by post. None were complaining of knee problems or awaiting revision surgery.

Of the 186 patients, there was a mean age of 66.9 years (26 to 87 ) at operation. The predominant pre-operative diagnosis was osteoarthritis, with only 23 patients having a diagnosis of rheumatoid arthritis.

The mean post-operative range of movement at final review in the 186 patients ( 192 knees) was $113^{\circ}$ ( $75^{\circ}$ to $\left.130^{\circ}\right)$. The posterior cruciate ligament was retained in 110 patients ( 110 knees) with meniscal bearing Low Contact Stress prostheses and sacrificed in 82 rotating platform Low Contact Stress implants. There were 122 cemented, 47 uncemented and 23 hybrid TKRs (cemented tibia, uncemented femur). The patella was not resurfaced in 17 patients (17 knees).

All the knee scores showed a highly significant improvement at one, three, seven and ten years compared with the pre-operative scores $(t$-test, $\mathrm{p}<0.001)$. The improved 
Table I. Mean knee scores at each follow-up interval. All scores significantly improved compared with pre-operative score $(t$-test, $\mathrm{p}<0.0001)$

\begin{tabular}{|c|c|c|c|c|c|}
\hline \multirow[b]{2}{*}{ Knee score $^{*}$ (SD) } & \multirow[b]{2}{*}{ Pre-operative } & \multicolumn{4}{|l|}{ Year } \\
\hline & & 1 & 3 & 7 & 10 \\
\hline KS function & $44.21(22.1)$ & $62.81(22.5)$ & $63.04(22.6)$ & $59.79(28.4)$ & \\
\hline KS pain & $36.64(17.4)$ & $82.47(13.5)$ & 87.45 (12.7) & $86.93(15.7)$ & \\
\hline NJKS & $60.51(11.8)$ & $82.32(10.2)$ & $84.22(10.2)$ & $83.34(12.8)$ & \\
\hline WOMAC & $54.84(16.6)$ & $19.26(17.7)$ & $18.34(16.5)$ & $25.55(18.5)$ & $27.56(20.7)$ \\
\hline
\end{tabular}

* KS, knee score; NJKS, New Jersey Knee score; WOMAC, Western Ontario McMasters University index

Table II. Mean knee scores comparing posterior cruciate retaining meniscal and posterior cruciate sacrificing rotating platform knee replacement

\begin{tabular}{lcccc}
\hline Knee score (SD) & KS $^{*}$ function & KS pain & NJKS $^{\dagger}$ & WOMAC $^{\ddagger}$ \\
\hline Low Contact Stress & & & & \\
Meniscal bearing & $60(28.0)$ & $87(14.3)$ & $84(12.6)$ & $18(17.6)$ \\
Rotating platform & $59(27.9)$ & $85(16.6)$ & $82(12.4)$ & $18(19.1)$ \\
\hline * KS, knee score & & & & \\
+ NJKS, New Jersey Knee score & & \\
‡ WOMAC, Western Ontario McMasters University index
\end{tabular}

scores at one and three years had marginally deteriorated by seven and ten years, but this was not significant $(t$-test, $\mathrm{p}=0.05)$ (Table I). There was no statistical difference $(t$ test, $\mathrm{p}=0.05$ ) between those who had an uncemented component and those having a hybrid or a cemented implant. The posterior cruciate ligament retaining group scored better with all knee scores, but this did not reach statistical significance (t-test, $\mathrm{p}>0.05$ )(Table II).

Survival analysis, using further revision for any reason, showed a 12-year survival of $92 \%$ (95\% CI 90 to 94) (Fig. 1). Survival curves using implant failure due to aseptic loosening showed an improved result, with a 12-year survival of $98 \%$ (95\% CI 97 to 99) (Fig. 2). When revision for aseptic loosening and polyethylene wear were combined, the 12-year survival decreased to $97 \%$ (95\% CI 94 to 96) (Fig. 3).

Radiological assessment. A total of 244 knees in 238 patients was assessed. A correlation analysis was undertaken between the WOMAC scores at ten years and the $\alpha, \Omega$ and $\gamma$ angles measured on the one-year post-operative radiograph in accordance with the Knee Society evaluation system. ${ }^{9}$ A significant correlation was found for all three angles $(\mathrm{p}<0.01)$ (Tables III and IV). A similar analysis between the New Jersey Knee score and these angles found a significant correlation with the $\Omega$ angle ( $\mathrm{p}<0.05$ ) (Table IV).

There was no significant difference between outcome scores and the amount of lateral displacement of the patella on the skyline radiograph ( $t$-test, $\mathrm{p}>0.005$ ).

Only four radiolucent lines were identified in four knees at ten years, with the majority occurring beneath the medial tibial plateau (zone 1). ${ }^{10}$ No radiolucent line progressed over the period of review, and none was greater than $1 \mathrm{~mm}$ in width. There were three patients (three knees) with small lytic defects at the seven-year radiological review; two were located beneath the anterior femoral flange and one beneath the medial tibial plateau. A total of 186 patients (192 knees) were assessed at this stage. All the lytic lesions occurred in meniscal-bearing knees, which remained under observation without any plan to proceed to a revision. There were eight meniscal-bearing knees in eight patients with evidence of polyethylene wear shown during manual measurement of the knee space, albeit minor in all cases. There was no observable wear in the rotating platform knees.

Revision procedures. Of the 25 revisions in 25 patients only four (four patients) were for aseptic loosening. One had loosening within a year of surgery, and at revision both femoral and tibial components were loose, but there was no sign of infection. The other three cases required revision at between seven and ten years all for tibial loosening.

Dislocation of the meniscal bearings occurred in three patients (three knees). The bearings were replaced and the knees remained stable following this single procedure. A fracture of the tibia occurred because of insufficiency or stress fracture of the medial tibial plateau in four patients (four knees) in the presence of a stable and normal-appearing tibial component. There were three deep infections and three revisions for unexplained pain, two of which were found to have a loose component (one femoral, one tibial) and have been analysed as part of the aseptic loosening group. One patient each required revision for a malaligned femoral component, patellar resurfacing in a previously unresurfaced patella and failure of tibial ingrowth in an uncemented component.

There were seven patients (seven knees), five with meniscal bearing, two with rotating platforms, who underwent bearing exchange for polyethylene wear between seven and 12 years after their initial procedure. None of these patients were found to have loosening of the femoral and tibial components, which were accordingly retained. The meniscal bearings generally wore on the peripheral medial and lateral margins articulating with the femoral component where the polyethylene was scalloped and thin, or on the superior surface. Gross back-side wear was uncommon unless the bearing had been subluxing in the tibial tray. 
Table III. Radiological results $\left(^{\circ}\right)$

\begin{tabular}{lccrc}
\hline & Minimum & Maximum & \multicolumn{2}{c}{ Mean (SD) } \\
\hline$\alpha$ angle & 87 & 113 & 96.43 & $(3.02)$ \\
$\beta$ angle & 82 & 93 & 88.63 & $(1.92)$ \\
$\Omega$ angle & 0 & 14 & 5.17 & $(2.82)$ \\
$\gamma$ angle & 0 & 10 & 3.16 & $(2.61)$ \\
$\sigma$ angle & 73 & 92 & 82.30 & $(3.02)$ \\
Patellar/patellar tendon ratio & 0 & 1.79 & 0.65 & $(0.26)$ \\
Patellar subluxation $(\mathrm{mm})$ & 0 & 8 & 1.92 & $(2.18)$ \\
\hline
\end{tabular}

Table IV. The radiological position of the components compared with the knee scores

\begin{tabular}{|c|c|c|c|c|}
\hline Radiological angle & Knee score pain & Knee score function & NJKS $^{*}$ & WOMAC $^{\dagger}$ \\
\hline \multicolumn{5}{|l|}{$\alpha$} \\
\hline Pearson's correlation & 0.090 & 0.028 & 0.090 & $-0.162^{\ddagger}$ \\
\hline Significance (2-tailed) & 0.126 & 0.635 & 0.123 & 0.006 \\
\hline \multicolumn{5}{|l|}{$\beta$} \\
\hline Pearson's correlation & 0.008 & -0.012 & 0.010 & 0.033 \\
\hline Significance (2-tailed) & 0.890 & 0.835 & 0.858 & 0.585 \\
\hline \multicolumn{5}{|l|}{$\Omega$} \\
\hline Pearson's correlation & 0.062 & 0.060 & 0.118 & $-0.170^{\ddagger}$ \\
\hline Significance (2-tailed) & 0.299 & 0.310 & 0.045 & 0.005 \\
\hline \multicolumn{5}{|l|}{$\gamma$} \\
\hline Pearson's correlation & 0.108 & 0.043 & 0.085 & $-0.166^{\ddagger}$ \\
\hline Significance (2-tailed) & 0.069 & 0.467 & 0.154 & 0.006 \\
\hline \multicolumn{5}{|l|}{$\Sigma$} \\
\hline Pearson's correlation & -0.021 & -0.041 & -0.066 & 0.059 \\
\hline Significance (2-tailed) & 0.723 & 0.494 & 0.267 & 0.330 \\
\hline
\end{tabular}

There was no difference comparing the one year radiological parameters between this group and the rest of the study group ( $\mathrm{t}$-test, $\mathrm{p}>0.005$ ).

\section{Discussion}

A recent biomechanical assessment of mobile-bearing Low Contact Stress TKR has confirmed the earlier assumption that increasing congruity and mobility decouples knee movement, resulting in mainly unidirectional movement at the femoral-polyethylene interface and subsequent reduction in volumetric wear. ${ }^{15,17}$ Clinical and radiological results with other mobile-bearing designs have also confirmed the low rate of wear and associated osteolysis. ${ }^{18,19}$ Initial concerns that a mobile polyethylene bearing might increase volumetric wear by adding a further articulating surface have not been substantiated, with most laboratory and retrieval studies reporting minimal back-side wear. ${ }^{20,21}$ Initial studies using serial plain radiographs ${ }^{22}$ and recently employing fluoroscopy ${ }^{23}$ have confirmed that the bearings in the Low Contact Stress TKR remain mobile, and conse- quently retain the ability to realign the polyethylene during knee function, with resultant reduction in volumetric wear.

The survival of our series at 12 years was 97\% (95\% CI 96 to 98 ), for revision due to wear and loosening, supporting our hypothesis that the wear rate would be low with this design, and were at least comparable with other studies using a fixedbearing TKR, ${ }^{1-6}$ and matched the experience of the designing surgeon. ${ }^{9}$ The incidence of radiolucent lines and lytic areas was also very small. However, eight knees had measurable early polyethylene wear, all were knees with a meniscal bearing, but all were functioning satisfactorily, with none due for revision. There was no measurable wear of the rotating platform component with the method used. Measurement of minor change in the thickness of the polyethylene component was difficult using the standard weight-bearing radiographs, as the posterior slope of the tibial component often resulted in the true height of the polyethylene bearing being obscured on the AP radiograph. We did not use cine-radiography or other radiological methods to obtain exposures with the beam absolutely parallel to the base plate. 


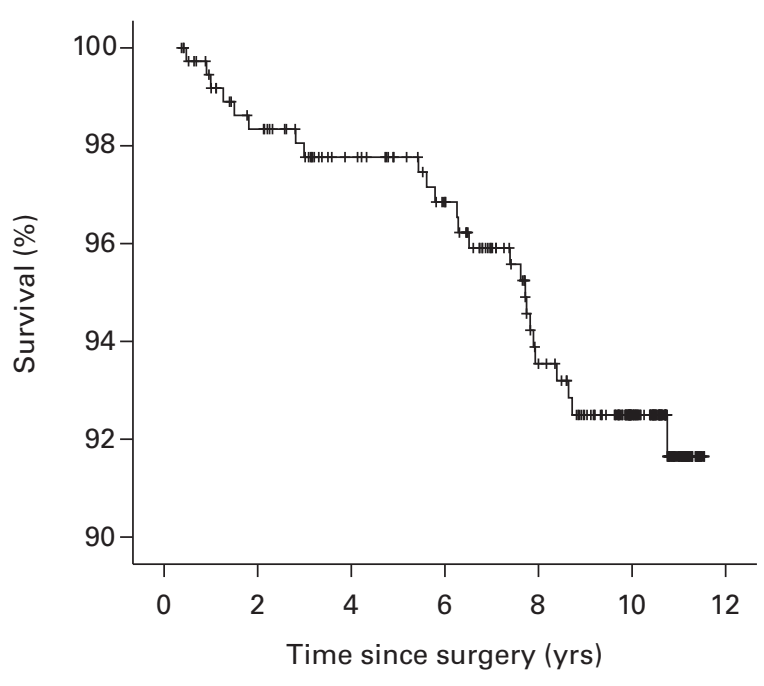

Fig. 1

Survival curve for the study group using revision for any cause as failure.

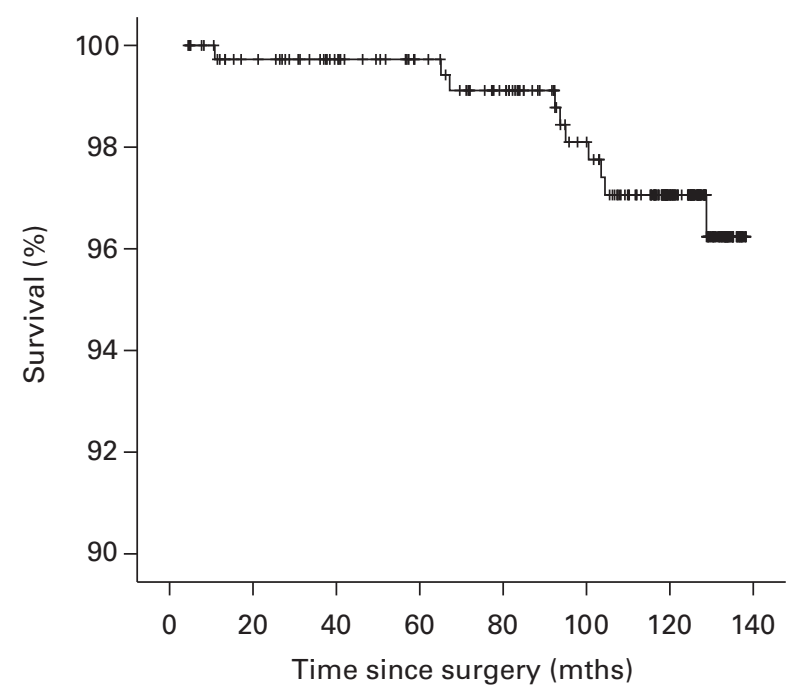

Fig. 2

Survival curve using revision for loosening and polyethylene wear as failure.

The polyethylene used during the course of this study was irradiated in air and had a variable shelf-life. These factors are now known to be related to polyethylene oxidation and subsequent wear of the insert. ${ }^{21,24}$ Another factor likely to have increased the polyethylene wear was the early design of the meniscal bearings, which had scalloped edges to increase the coronal stability. These margins were very thin, and at revision it was often observed that the majority of the wear occurred at these sites. These findings were not observed with the rotating platform, and have not been reported in long-term studies using this implant. ${ }^{25,30}$ Changes made to the meniscal bearings in the mid-1990s

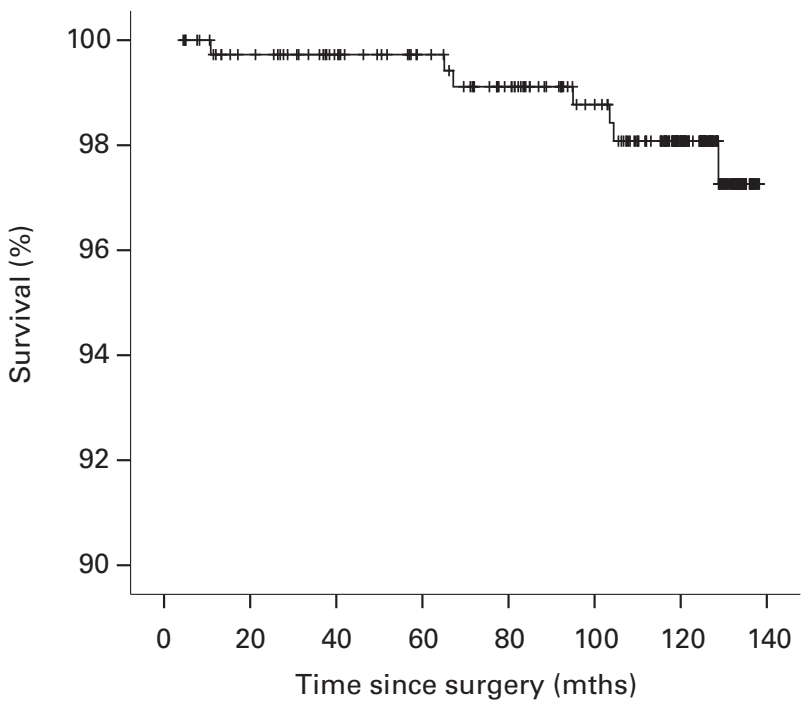

Fig. 3

Survival curve using revision due to polyethylene wear as failure.

reduced the thin edge and the edge loading associated with the wear observed at revision surgery.

Early revision procedures were performed for a variety of reasons, including unexplained pain and stiffness. Such problems are not specifically related to a single implant design. Malposition of components after surgery has been associated with poorer survival rates.31,32 However, we could find no correlation between the position of the components after one year and the need for revision for any cause. There was a correlation between the overall mechanical axis and the WOMAC score, which adds support to the assertion that the component position affects the patient's function.

Although the meniscal-bearing implant, which retains the posterior cruciate ligament achieved better knee scores than the rotating platform, which sacrifices this structure, the results were not statistically significants. The difference may be due to a tendency to use the meniscal-bearing implant in younger patients with less severe disease.

In this series it was our practice to resurface the patella in most cases. There were no cases of patellar failure and the mobile bearing of the patella appeared to function satisfactorily even in cases with subluxation $>5 \mathrm{~mm}$. Although it was our practice to revise the patellar mobile-bearing at the same time when other polyethylene bearings were replaced, no knee underwent revision solely for patellar wear or loosening. Indeed at the time of revision the patellar bearing often looked normal, reflecting the findings of other studies suggesting that the Low Contact Stress patellar component is well tolerated. ${ }^{14,25}$

This study had significant problems with follow-up because of the death rate and the high incidence of comorbidities. The New Zealand Joint Registry helped in validating our figures, but we accept that it does not take 
into account those who have left the country. Of the 24 patients who were lost to follow-up, at least 12 were known to have emigrated.

This study is unique in that it represents the collective experience of 14 general orthopaedic surgeons serving a single city, all of whom used the Low Contact Stress mobile-bearing TKR as the only prosthesis for all primary TKRs. As only three revision procedures were undertaken for dislocation of the bearing it suggests that this prosthesis is reliable in virtually all primary situations, irrespective of the pre-operative deformity or pathology.

The authors wish to thank the Christchurch Orthopaedic Group for their participation in this study and Mrs T. Hobbs, Research assistant, for the collection of patient data.

No benefits in any form have been received or will be received from a com mercial party related directly or indirectly to the subject of this article.

\section{References}

1. Gill GS, Joshi AB. Long-term results of Kinematic Condylar knee replacement: an analysis of 404 knees. J Bone Joint Surg [Br] 2001;83-B:355-8.

2. Vessely MB, Whaley AL, Harmsen WS, Schleck CD, Berry DJ. Long term survivorship and failure modes of 1000 cemented condylar total knee arthroplasties. Clin Orthop 2006;452:28-34.

3. Rodricks DJ, Patil S, Pulido P, Colwell CW Jr. Press-fit condylar design total knee arthroplasty: fourteen to seventeen-year follow-up. J Bone Joint Surg [Am] 2007;89-A:89-95

4. Epinette JA, Manley MT. Hydroxyapatite-coated total knee replacement: clinical experience at 10 to 15 years. J Bone Joint Surg [Br] 2007;89-B:34-8.

5. Baker PN, Khaw FM, Kirk LMG, Elser CNA, Gregg PJ. A randomised controlled trial of cemented versus cementless press-fit condylar total knee replacement: 15 year survival analysis. J Bone Joint Surg [Br] 2007;89-B:1608-14.

6. Kim YH, Yoon SH, Kim JS. The long-term results of simultaneous fixed-bearing and mobile-bearing total knee replacements performed in the same patient. J Bone Joint Surg [Br] 2007;89-B:1317-23

7. Evison JC, Hooper GJ. Kinematic total knee arthroplasty. J Bone Joint Surg [Br] 1993;75-B(Suppl I):49.

8. Abernathy PJ, Robinson CM, Fowler RM. Fracture of the metal tibia tray after kinematic total knee replacement: a common cause of early aseptic failure. J Bone Joint Surg [Br] 1996;78-B:220-5.

9. Buechel FF, Buechel FF Jr, Pappas MJ, D'Alessio J. Twenty-year evaluation of meniscal bearing and rotating platform knee replacements. Clin Orthop 2001;388:41-50

10. Insall JN, Dorr LD, Scott RD, Scott WN. Rationale of the Knee Society clinical rating system. Clin Orthop 1989;248:13-14.

11. Buechel FF. A simplified evaluation system for the rating of knee function. Orthop Rev 1982;11:97-101.
12. Bellamy N, Buchanan WW, Goldsmith CH, Campbell J, Stitt LW. Validation study of WOMAC: a health status instrument for measuring clinically important patient relevant outcomes to antirheumatic drug therapy in patients with osteoarthritis of the hip or knee. J Rheumatol 1988;15:1833-40.

13. Ewald FC. The Knee Society total knee arthroplasty roentgenographic evaluation and scoring system. Clin Orthop 1989;248:9-12.

14. Hooper GJ. Patella complications. In: Hamelynck KJ, Stiehl JB, eds. LCS mobile bearing arthroplasty: 25 years worldwide experience. Heidelberg: Springer, 2002:260-3.

15. Kaplan EL, Meier P. Nonparametric estimation from incomplete observations. J Am Stat Assn 1958;53:457-81.

16. No authors listed. New Zealand Joint Registry, New Zealand Orthopaedic Association. http://www.cdhb.govt.nz/njr (date last accessed 27 October 2008).

17. McEwen HM, Fisher J, Goldsmith AA, et al. Wear of fixed bearing and rotating platform mobile bearing knees subjected to high levels of internal and external tibial rotation. J Mater Sci Mater Med 2001;12:1049-52.

18. Kim YH, Kim JS. Comparison of anterior-posterior-glide and rotating-platform low contact stress mobile-bearing total knee arthroplasties. J Bone Joint Surg [Am] 2004;86-A:1236-47.

19. Ho FY, Ma HM, Liau JJ, Yeh CR, Huang CH. Mobile-bearing knees reduce rotational asymmetric wear. Clin Orthop 2007;463:143-9.

20. Kop AM, Swarts E. Quantification of polyethylene degradation in mobile bearing knees: a retrieval analysis of the anterior-posterior glide (APG) and rotating platform (RP) and Low Contact Stress (LCS) knee. Acta Orthop 2007;78:364-70.

21. Collier MB, Engh CA Jr, McAuley JP, Engh GA. Factors associated with the loss of thickness of polyethylene tibial bearings after knee arthroplasty. J Bone Joint Surg [Am]2007;89-A:1306-14

22. Maxwell AR, Hooper GJ, Jones DI, Penny ID. Meniscal bearing motion in the LCS total knee replacement. J Bone Joint Surg [Br] 1992;74-B(Suppl):42.

23. Stiehl JB, Dennis DA, Komistek RD, Keblish PA. Kinematic analysis of a mobile bearing total knee arthroplasty. Clin Orthop 1997;345:60-5.

24. Williams IR, Mayor MB, Collier JP. Impact of sterilization method on wear in knee arthroplasty. Clin Orthop 1998;356:170-80.

25. Callaghan JJ, O'Rourke MR, lossi MF, et al. Cemented rotating-platform total knee replacement: a concise follow-up at a minimum of fifteen years, of a previous report. J Bone Joint Surg [Am] 2005;87-A:1995-8.

26. Sorrells RB, Capps SG. Clinical results of primary low contact stress cementless total knee arthroplasty. Orthopedics 2006;29(Suppl 9):42-4.

27. Sansone V, da Gama Malcher M. Mobile bearing total knee prosthesis: a 5 to 9 year follow-up of the first 110 consecutive arthroplasties. J Arthroplasty 2004;19:678-85.

28. Sorrels RB. Primary knee arthroplasty: long-term outcomes, the rotating platform mobile bearing TKA. Orthopedics 1996; 199:793-96.

29. Jordan LR, Olivio JL, Voorhorst PE. Survivorship analysis of cementless meniscal bearing total knee arthroplasty. Clin Orthop 1997;338:119-23.

30. Callaghan JJ, Squire MW, Goetz DD, Sullivan PM, Johnston RC. Cemented rotating platform total knee replacement: a nine to twelve-year follow-up study. $J$ Bone Joint Surg [Am] 2000;82-A:705-11.

31. Davis CR, Davies AP, Newman JH. Cases of failure of the Kinemax Plus total knee replacement in the first five years. Knee 2007;14:158-61.

32. Windsor RE, Scuderi GR, Moran MC, Insall JN. Mechanism of failure of the femoral and tibial components in total knee arthroplasty. Clin Orthop 1989;248:15-19. 